\title{
Studying time-like electromagnetic baryonic transitions with HADES in pion induced reactions
}

\author{
Béatrice Ramstein ${ }^{1, *}$ \\ for the HADES collaboration \\ ${ }^{1}$ Institut de Physique Nucléaire d'Orsay, CNRS-IN2P3, Univ. Paris Sud, Univ. Paris-Saclay, 91406 Orsay Cedex, France.
}

\begin{abstract}
A dedicated programme aiming at studying electromagnetic baryonic transitions in the time-like region has started at GSI using the pion beam and the High Acceptance Di-Electron Spectrometer (HADES) set-up. A pioneering experiment has been carried out in the second resonance region, at a center-of-mass energy of $1.49 \mathrm{GeV}$, using carbon and polyethylene targets, allowing for an analysis of the inclusive $e^{+} e^{-}$production and of the exclusive quasi-free $\pi^{-} p \rightarrow n e^{+} e^{-}$reaction, in complement to hadronic channels. Predictions for the Dalitz decay of $\mathrm{N}(1520)$ and $\mathrm{N}(1535)$ based on real photon couplings strongly underestimate the $e^{+} e^{-}$yield at invariant masses larger than $300 \mathrm{MeV} / \mathrm{c}^{2}$, which signals effects of time-like baryon transition form factors, in qualitative agreement with the Vector Dominance Models (VDM). A quantitative description of the observed $e^{+} e^{-}$yield is achieved by taking into account the contribution from off-shell $\rho$ s. The latter was provided by the preliminary results of a Bonn-Gatchina Partial Wave Analysis of the two-pion production channels, which were measured simultaneously in our experiment. A good agreement is also obtained using a covariant model for the time-like electromagnetic form factors for the $\mathrm{N}-\mathrm{N}(1520)$ transition. The angular distributions for the $e^{+} e^{-}$production contain additional selective information on the spin structure of the different transitions. The measurements with the pion beam will be extended in future in the third resonance region. Hyperon Dalitz decay studies in proton induced reactions are also foreseen.
\end{abstract}

\section{Introduction}

Dilepton production is a favored probe for QCD matter studies. In particular, the emission of low mass dileptons gives insight on the properties of vector mesons in the hadronic matter. It allows to search for in-medium modifications of the $\rho$ mesons [1], as predicted by hadronic many-body calculations [1] due to the dressing of the pions and the direct coupling of the $\rho$ meson to baryons. These spectral functions are also expected to be affected by QCD chiral symmetry restoration in hot and dense matter. The main objective of experiments with the High Acceptance Di-Electron Spectrometer (HADES) [2] at GSI is the study of hadronic matter in the low temperature $(\mathrm{T}<$ $80 \mathrm{MeV}$ ) and large density (up to 3 times the normal nuclear matter density) region [3]. An experimental program using $p p$, "quasifree" $n p$ and more recently $\pi N$ reactions is also developped, in order to provide reference spectra needed for the interpretation of medium effects and to isolate specific processes using exclusive channels, notably the baryon resonance Dalitz decays $\left(B \rightarrow N e^{+} e^{-}\right)$. An overview of HADES results is given in P. Salabura's report at this conference [4] and preliminary results concerning the hadronic channels measured in $\pi^{-} p$ collisions in the second resonance region can be found in the contribution of I. Ciepał to this conference [5]. This contribution is de-

*e-mail: ramstein@ipno.in2p3.fr voted to the study of electromagnetic channels measured with the pion beam in the same energy range.

\section{Dalitz decay studies with HADES}

Baryon resonance Dalitz decays $\left(B \rightarrow N e^{+} e^{-}\right.$) give insight into the electromagnetic structure of baryonic transitions for squared momentum transfer $q^{2}=M_{e e}^{2}$ in the time-like region $\left(q^{2}>0\right)$, in the $\left[4 m_{e}^{2},\left(M_{B}-M_{N}\right)^{2}\right]$ range, where $M_{e e}$ is the $e^{+} e^{-}$invariant mass, $m_{e}, M_{B}$ and $M_{N}$ are the electron, baryon resonance and nucleon masses, respectively. As illustrated in figure. 1, this kinematical region is complementary to the space-like region $\left(q^{2}<0\right)$ probed in electron scattering, in particular with the CLAS detector at JLab [6]. The two regions are connected at the "photon point" $\left(q^{2}=0\right)$, where information on the $\mathrm{N}-\gamma$ couplings is provided by meson photoproduction experiments. For larger positive $q^{2}$, the influence of the $\rho$ and $\omega$ vector mesons is expected to play a significant role. Dalitz decay processes are therefore particularly relevant to investigate the applicability of Vector Dominance Model (VDM) in electromagnetic baryon transitions. HADES measurements in proton-proton collisions at an incident energy of $1.25 \mathrm{GeV}$ provided the first determination of the $\Delta(1232)$ Dalitz decay branching ratio $[7,8]$ in the channel $p p \rightarrow p p e^{+} e^{-}$. At higher incident proton energies, the $e^{+} e^{-}$invariant mass spectra clearly demonstrate the effect of electromagnetic form factors of VDM type [9-11], which means that the 
Time-Like electromagnetic form factors

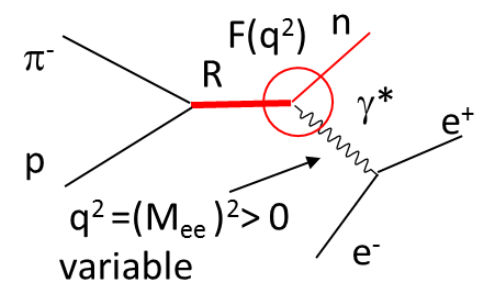

Space-Like electromagnetic form factors

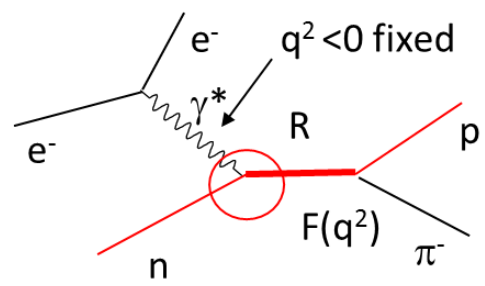

Figure 1. Left: Sketch of the Dalitz decay process of a resonance produced in the s-channel in a $\pi^{-} p$ reaction. The four-momentum transfer $q^{2}$ is positive and takes values between $4 m_{e}^{2}$ and $\left(M_{B}-M_{N}\right)^{2}$, where $M_{e e}$ is the $e^{+} e^{-}$invariant mass, $m_{e}, M_{B}$ and $M_{N}$ are the electron, baryon resonance and nucleon masses, respectively. Right: Excitation of a nucleon by electron scattering. The fourmomentum transfer $q^{2}$ is negative and fixed by the energy and angle of the scattered electron.

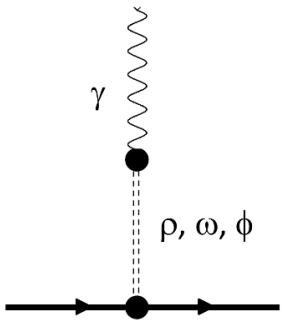

Figure 2. Sketch of the Vector Dominance Model. The electromagnetic interaction is mediated by vector mesons.

electromagnetic interaction is mediated by vector mesons as illustrated in figure 2. As discussed in [4], the results obtained in $p p$ and $p n$ reactions have been crucial to provide a reference for the study of off-shell $\rho$ propagation in cold as well as hot and dense matter.

The extension of the program to experiments with the GSI pion beam offers a more direct way to investigate Dalitz decays. In $\pi N$ reactions the baryon resonances are indeed produced with a fixed mass in the s channel $\left(M_{B}=\sqrt{s}\right)$, so there is less overlap between several states and the $\pi N$ interaction is better controlled. In addition, the exclusive channel $\pi^{-} \mathrm{p} \rightarrow \mathrm{n} e^{+} e^{-}$can be studied by selecting $e^{+} e^{-}$pairs in a missing mass range around the neutron mass, while in the case of the $p p$ reaction, the request of a detected proton significantly reduces the detection acceptance in HADES. These reactions are in fact the reverse reaction from the pion electroproduction reaction, which is one of the experiments used to extract baryon transition form factors in the space-like region (see figure 1).

\section{The pion beam line and the Hades detector}

High Acceptance Di-Electron Spectrometer (HADES) is a versatile set-up [2] installed at SIS18 in GSI, Darmstadt. It provides excellent detection both for hadron and rare dielectron production, thanks to the high acceptance $(30 \%$ for $e^{+} e^{-}$pairs in the vector meson region), the powerful particle $(p / K / \pi / e)$ identification and the very good mass resolution ( 2 - 3\% for $\omega \rightarrow e^{+} e^{-}$decays). Pion beam experiments were since the very beginning part of the HADES scientific program and commissioning runs took place already in 1999 [12, 13]. To check the pion beam properties, a test experiment using the HADES detector was performed in 2014. The secondary pion beam is produced using an extracted primary ${ }^{14} \mathrm{~N}$ beam provided by the SIS18 synchrotron with an intensity of $(0.8-1.0) \times$ $10^{11}$ ions/spill, close to the space-charge limit of the machine impinging on a $10 \mathrm{~cm}$ long beryllium target. The pions in a selected momentum range are transported through the beam line consisting of nine quadrupole and two dipole magnets from the production point up to the HADES target located $33 \mathrm{~m}$ downstream. The pion yield at the exit of the pion beam line reaches a maximum of about $10^{6}$ pions/spill at $p=1.0 \mathrm{GeV} / \mathrm{c}$ and is about twice smaller at $\mathrm{p}=0.7 \mathrm{GeV} / \mathrm{c}$ and $1.3 \mathrm{GeV} / \mathrm{c}$.

Two silicon strip detectors along the beam line and a start detector right in front of HADES were used to measure the position of the beam particles. Beam transport calculations have been performed to optimize the transmission of the beam line and reconstruct event-by-event the pion momentum with a precision better than $0.3 \%(\sigma)$.

\section{Data analysis}

Data at four different pion beam momenta $(0.654,0.686$, 0.738 and $0.787 \mathrm{GeV} / \mathrm{c})$ in the second resonance region were collected on both polyethylene $\left(\left(\mathrm{C}_{2} \mathrm{H}_{4}\right)_{n}\right)$ and carbon targets. The largest statistics was measured in the case of $0.69 \mathrm{GeV} / \mathrm{c}$ on the polyethylene target and this data set was used for dilepton production studies. As described in detail in [5], the precise normalization of the measurements is provided by the known $\pi^{-}$p elastic scattering differential cross sections [14], after subtraction of the carbon contribution, using a least-square tagging procedure, based on the comparison of measurements with the polyethylene and carbon targets. The data measured for the exclusive $\pi^{-} p \rightarrow \pi^{+} \pi^{-} n$ and $\pi^{-} p \rightarrow \pi^{-} \pi^{0} p$ reactions have 


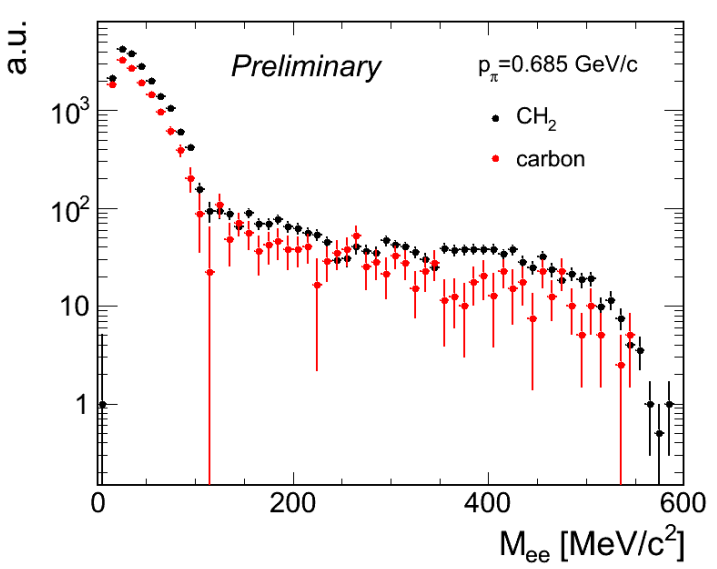

Figure 3. $e^{+} e^{-}$invariant mass distributions measured in the interaction of pions at $\mathrm{p}=0.685 \mathrm{GeV} / \mathrm{c}$ on polyethylene (black dots) and carbon (red dots) after normalization to the same incident flux.

then been included in the multichannel Partial Wave Analysis (PWA) developed by the Bonn-Gatchina group [15]. In this way, the different contributions to the total amplitude, such as $\Delta \pi, \rho \mathrm{N}, \sigma \mathrm{N}$ can be extracted together with their couplings to the main contributing baryon resonances $(\mathrm{N}(1520), \mathrm{N}(1535), \mathrm{N}(1440), \ldots)$. The $\rho \mathrm{N}$ contribution deduced from this analysis is used as an input for the description of the dielectron channels, as described in sec. 5. For the $e^{+} e^{-}$channel, the subtraction of events produced by the interaction of the pion beam with carbon nuclei in the polyethylene target was not feasible, due to the too small statistics recorded on the carbon target. Specific analysis cuts were applied for the reconstruction of correlated $e^{+} e^{-}$pairs, in order to suppress tracks arising from photon conversion and to avoid their contribution to the combinatorial background [16]. The combinatorial background is then estimated using the like-sign pair yields measured in the same event. A threshold of $9^{\circ}$ applied to the opening angle between the two leptons allowed for the complete rejection of correlated pairs from the photon conversion.

\section{$5 e^{+} e^{-}$invariant mass and missing mass studies}

The $e^{+} e^{-}$invariant mass distributions are displayed in figure 3 for both the carbon (red dots) and the polyethylene (black dots) targets after normalization to the same incident yields. The contribution due to interactions with the carbon nuclei is on average $70 \%$ for invariant masses below $300 \mathrm{MeV} / \mathrm{c}^{2}$ and $50 \%$ above.

For $e^{+} e^{-}$invariant masses above the $\pi^{0}$ mass, the $\pi^{0}$ Dalitz decay does not contribute and the missing mass distribution clearly shows two structures (figure 4). The peak around the neutron mass can be attributed to the exclusive $\pi^{-} \mathrm{p} \rightarrow \mathrm{n} e^{+} e^{-}$reaction, either on a free proton or a bound proton in the carbon nuclei, in a quasi-free process. The contribution at larger missing masses is expected to be mainly due to the $\eta$ Dalitz decay, where a photon is

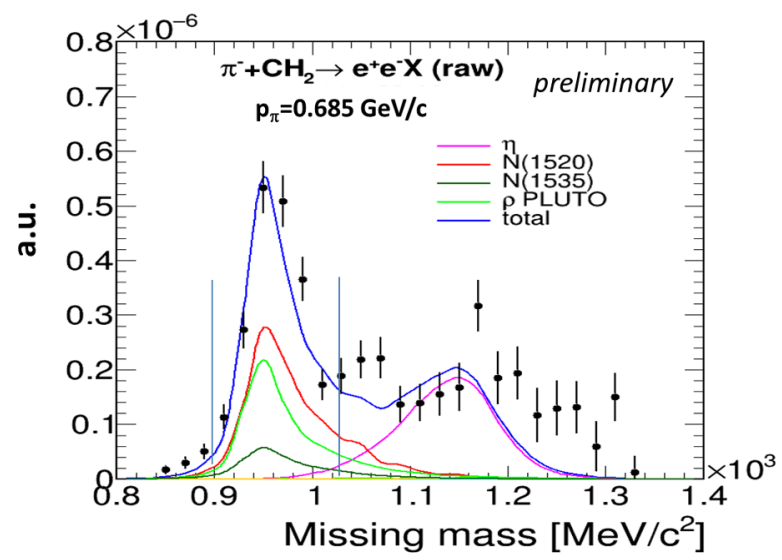

Figure 4. Distribution of the missing mass of $e^{+} e^{-}$events with invariant mass larger than $120 \mathrm{MeV} / \mathrm{c}^{2}$. The results of the simulation (total shown as a blue curve) including the $\eta$ (pink), N(1520) Dalitz decay (red), N(1535) Dalitz decay (dark green), $\rho$ (light green) are compared to the simulation showing the different components.

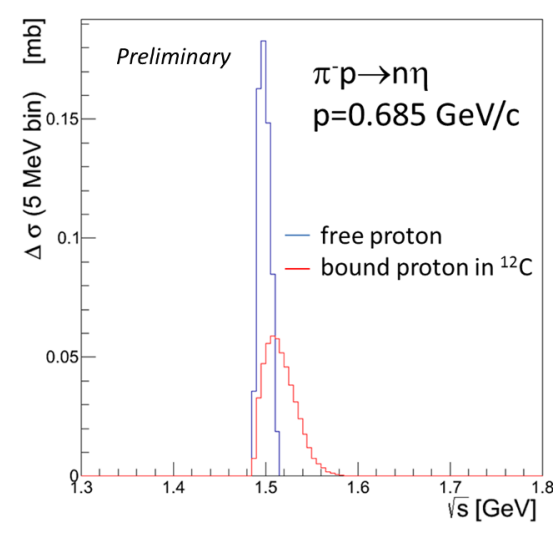

Figure 5. Distribution of cross sections for the $\pi^{-} p \rightarrow \eta n$ reaction in $5 \mathrm{MeV}$ wide bins in $\sqrt{s}$ (center-of-mass energy) for the interaction with a free proton (blue) and a bound proton in the carbon target (red).

emitted in addition to the $e^{+} e^{-}$pair. The exclusive free or quasi-free $\pi^{-} \mathrm{p} \rightarrow \mathrm{n} e^{+} e^{-}$channel can then be selected by applying invariant mass $\left(\mathrm{M}_{e e}>0.140 \mathrm{GeV} / \mathrm{c}^{2}\right)$ and missing mass $\left(0.9<\mathrm{MM}<1.03 \mathrm{GeV} / \mathrm{c}^{2}\right)$ cuts, as shown on figure 4 . The number of selected events amounts to about 1500 .

To describe the $e^{+} e^{-}$production in $\pi^{-}+\left(\mathrm{C}_{2} \mathrm{H}_{4}\right)_{n}$ reactions, we developed simulations with the Pluto [17] event generator based on the available information for pionnucleon interactions. For the $\pi^{0}$ production cross sections in the channels $\pi^{-} p \rightarrow \pi^{0} n, \pi^{-} p \rightarrow \pi^{0} \pi^{0} n$ and $\pi^{-} p \rightarrow$ $\pi^{0} \pi^{-} p$, we use information from former data and from the SAID Partial Wave Analysis [14, 18-22]. The rapid variation of the $\eta$ production cross section close to threshold has also been measured [23]. An essential ingredient of the simulation of the $\pi^{-} p$ reactions is the pion beam momentum distribution, which is known from the pion beam 


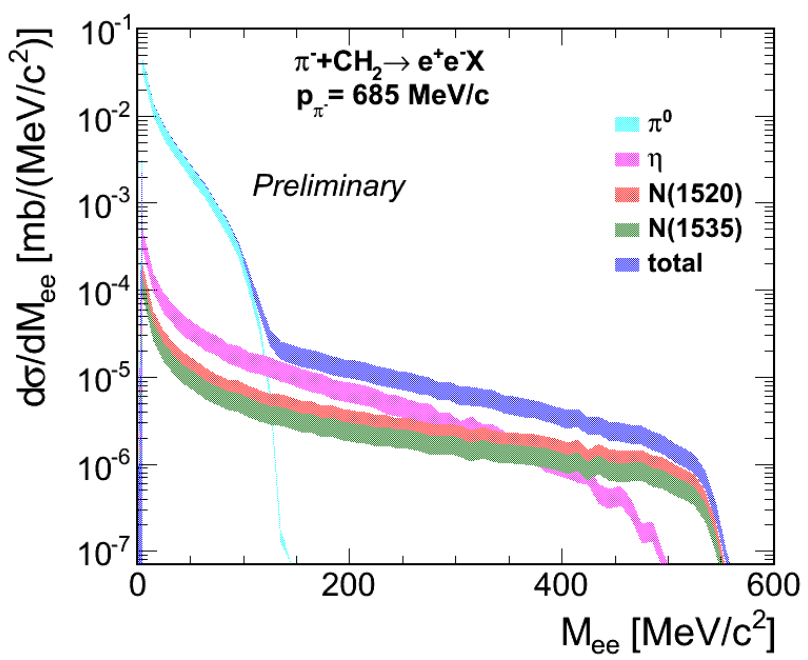

Figure 6. Differential cross sections as a function of the $e^{+} e^{-}$ invariant mass for the different contributions used as inputs for the simulations of the $\pi^{-} \mathrm{p} \rightarrow \mathrm{n} e^{+} e^{-} \mathrm{X}$ reaction at an incident pion momentum of $0.685 \mathrm{GeV} / \mathrm{c}$.

momentum reconstruction deduced from measured hits in the pion beam tracker using the optic parameters of the pion beam line. This distribution is centered at a value ( $\mathrm{p}=0.686 \mathrm{GeV} / \mathrm{c}$ ) very close to the $\eta$ production threshold and can be approximated by a gaussian with a width of $1.7 \%$. The $\eta$ yield resulting from the folding of the $\eta$ production excitation function and the beam momentum distribution is displayed in figure 5. The $e^{+} e^{-}$invariant mass distributions for the meson Dalitz decays are then deduced from a QED calculation using VDM form factors [24]. In addition to the Dalitz decay of the $\pi^{0}$ and $\eta$ mesons, we can expect production of $e^{+} e^{-}$via the Dalitz decays of baryons. It is known from the PWA analysis of the two pion channels that the dominant resonant contributions in the $s$ channel are due to $\mathrm{N}(1520)$ and $\mathrm{N}(1535)$ [5]. Starting from the QED calculation of [25], we found that the widths for the decay of the N(1520) $\left(\mathrm{J}^{P}=3 / 2^{-}\right)$and $\mathrm{N}(1535)\left(\mathrm{J}^{P}=1 / 2^{-}\right)$into a nucleon and a virtual photon of mass $M_{e e}$ can be deduced from the corresponding radiative decay widths by the same relation:

$$
\begin{aligned}
\Gamma^{N^{\star} \rightarrow N \gamma^{*}}\left(q^{2}\right) & =\frac{\sigma_{+}^{3} \sigma_{-}}{m_{+}^{3} m_{-}} \frac{\left|G_{T}\left(q^{2}\right)\right|^{2}}{\left|G_{T}(0)\right|^{2}} \times \Gamma^{N^{*} \rightarrow N \gamma} \\
\text { where } q^{2} & =M_{e e}^{2} \\
m_{ \pm} & =m_{\star} \pm m_{N}, \\
\sigma_{ \pm}^{2} & =m_{ \pm}^{2}-M_{e e}^{2},
\end{aligned}
$$

$m_{\star}$ is the resonance mass, $m_{N}$ is the nucleon mass and $G_{T}\left(q^{2}\right)$ is an effective form factor, which can be expressed as a function of the electric, magnetic and Coulomb form factors. The Dalitz decay differential decay width can then be calculated as a function of the $e^{+} e^{-}$invariant mass as

$$
\frac{d \Gamma^{N^{\star} \rightarrow N e^{+} e^{-}}}{d M_{e e}}=\frac{2 \alpha}{3 \pi M_{e e}} \Gamma_{M_{N^{\star}}^{N^{\star}} \rightarrow N \gamma^{*}}\left(M_{e e}\right),
$$

Assuming no dependence of the form factor on the four momentum transfer (point-like assumption), the differential Dalitz decay width of N(1520) and N(1535) can then be derived directly from the radiative decay width using Eq.1. To deduce the coresponding $e^{+} e^{-}$differential cross sections, we used the cross sections for the s-channel $\mathrm{N}(1520)$ and $\mathrm{N}(1535)$ resonant contributions in the $\pi^{-} p \rightarrow$ $n \gamma$ reaction, which were deduced, using detailed balance, from the Bonn-Gatchina PWA of the $\gamma n \rightarrow \pi^{-} p$ channel. We also implemented the Dalitz decay of the $\Delta(1232)$, using the $\Delta \pi$ cross sections extracted from the PWA of the two pion production channels in the $\pi^{-} \mathrm{p}$ reaction at our energy. The partial decay width was calculated using a constant form factor $\left(\mathrm{G}_{M}=3, \mathrm{G}_{E}=0, \mathrm{G}_{C}=0\right)$ consistent with the radiative decay width [25].

Interactions with carbon nuclei were treated in a quasifree approach, using the participant-spectator model and taking into account the nucleon momentum distribution in carbon. The available energy in the $\pi^{-} \mathrm{p}$ system, and hence the distribution of $\eta$ yields is quite sensitive to this assumption (figure 5). For the different contributions, the cross sections for interaction of $\pi^{-}$beam particles with carbon nuclei $\left(\sigma_{C}\right)$ were renormalized from the free cross sections $\left(s \sigma_{H}\right)$ taking into account the ratio between yields measured respectively in the $\left(\mathrm{C}_{2} \mathrm{H}_{4}\right)_{n}$ and $C$ targets. The total experimental and simulated yields are normalized as $\sigma_{H}+0.5 \sigma_{C}$ to take into account the different number of carbon and hydrogen atoms in polyethylene. The contributions of the different dilepton channels are then summed up incoherently. We consider two types of uncertainties to this dilepton cocktail. The first one is related to the knowledge of the free cross sections and the second one is due to the uncertainty of the carbon contribution. The cross section inputs for the simulation and the branching ratios to the $e^{+} e^{-}$emission are summarized in table 1 . Figure 6 shows the results of the simulation for the full solid angle, with uncertainties on each component displayed as colored bands. The spectrum is strongly dominated by the $\pi^{0}$ Dalitz decay for $e^{+} e^{-}$invariant masses below $120 \mathrm{MeV} / \mathrm{c}^{2}$, then the dominant contribution is the $\eta$ Dalitz, up to invariant masses of about $280 \mathrm{MeV} / \mathrm{c}^{2}$. For larger invariant masses, the Dalitz decay of N(1520) and $\mathrm{N}(1535)$ produced in the s-channel is found to be dominant. The $\Delta(1232)$ Dalitz decay contribution, which is about one order of magnitude lower than the N(1520) contribution and has a much steeper decrease as a function of $e^{+} e^{-}$invariant mass is not represented.

This cocktail can be considered as a reference for meson and baryon Dalitz decays in a point-like approach. It provides a reasonable description of the mesured dielectron yields for invariant masses below $300 \mathrm{MeV} / \mathrm{c}^{2}$, where the $\pi^{0}$ and $\eta$ Dalitz decay contributions dominate. However, it significantly underestimates the yield at higher invariant masses, where the baryon contribution is expected to take over. This deviation from a point-like behavior is even more striking in the exclusive $\pi^{-} \mathrm{p} \rightarrow n e^{+} e^{-}$channel, which clearly shows that time-like electromagnetic form factors need to be implemented. This was done in two different ways. 


\begin{tabular}{|c|c|c|c|c|}
\hline \multirow{2}{*}{ channel } & \multicolumn{3}{|c|}{$\sigma(\mathrm{mb})$} & \multirow{2}{*}{ Branching ratios } \\
\cline { 2 - 4 } & $\sigma_{H}$ & $\sigma_{C}$ & $\sigma_{H}+0.5 \times \sigma_{C}$ & \\
\hline$\pi^{0} \mathrm{n}$ & $9.0 \pm 0.8$ & $32.4 \pm 2.9 \pm 8.1$ & $25.2 \pm 2.3 \pm 4.1$ & \multirow{2}{*}{$\pi^{0} \rightarrow \gamma e^{+} e^{-} 1.2 \%$} \\
\hline$\pi^{0} \pi^{0} \mathrm{n}$ & $1.9 \pm 0.1$ & $6.8 \pm 0.4 \pm 1.7$ & $5.3 \pm 0.3 \pm 0.85$ & \\
\hline$\pi^{-} \pi^{0} \mathrm{p}$ & $4.0 \pm 0.5$ & $14.4 \pm 1.8 \pm 3.6$ & $11.2 \pm 1.4 \pm 1.8$ & \\
\hline$\eta \mathrm{n}$ & $0.63 \pm 0.2$ & $2.3 \pm 0.1 \pm 0.8$ & $1.8 \pm 0.25 \pm 0.4$ & $\eta \rightarrow \gamma e^{+} e^{-} 6.910^{-3}$ \\
\hline$\Delta^{+} \pm$ & $0.78 \pm 0.08$ & $2.9 \pm 0.3 \pm 1.0$ & $2.2 \pm 0.23 \pm 0.5$ & \multirow{2}{*}{$\Delta(1232) \rightarrow N e^{+} e^{-} 4.210^{-5}$} \\
\hline$\Delta^{0} \pi^{0}$ & $1 . \pm 0.1$ & $3.7 \pm 0.4 \pm 1.3$ & $2.9 \pm 0.3 \pm 0.65$ & \\
\hline $\mathrm{N}(1520)$ & $20.4 \pm 2$ & $75.5 \pm 7 \pm 27$ & $58 \pm 5.5 \pm 13.5$ & $N(1520)^{0} \rightarrow n e^{+} e^{-} 2.310^{-5}$ \\
\hline $\mathrm{N}(1535)$ & $9.7 \pm 1$ & $35.9 \pm 3.7 \pm 5.0$ & $28 \pm 2.9 \pm 2.5$ & $N(1535)^{0} \rightarrow n e^{+} e^{-} 3.510^{-5}$ \\
\hline
\end{tabular}

Table 1. Cross sections for the different channels implemented in the simulation for interactions of $\pi^{-}$with incident momentum of $0.685 \mathrm{GeV} / \mathrm{c}$ with proton, carbon and polyethylene targets. For the carbon and polyethylene targets, the first uncertainty is related to the elementary cross sections used in the participant-spectator approach, as given in the first column and the second to the ratio of carbon to proton cross section. The last column gives the branching ratio to the $e^{+} e^{-}$channel.

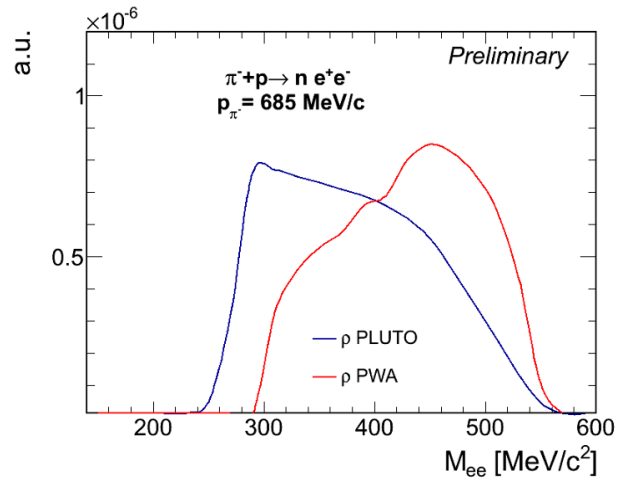

Figure 7. Simulations of the $e^{+} e^{-}$invariant mass distribution of the $\rho$ decay contribution to the $\pi^{-} \mathrm{p} \rightarrow \mathrm{n} e^{+} e^{-}$at $0.685 \mathrm{GeV} / \mathrm{c}$ with mass distributions from a Breit-Wigner (blue curve, labeled $\rho$ PLUTO) or deduced from the PWA of hadronic channels (red curve, labeled $\rho$ PWA).

The first approach was based on the VDM and took advantage of the simultaneous measurement of pionic channels. We used the off-shell $\rho$ contribution from the PWA in the $\pi^{-} p$ reaction at an incident momentum of $0.69 \mathrm{GeV} / \mathrm{c}$ and converted it into a dilepton contribution, using VDM. It has to be noted that the spectral shape of this off-shell $\rho$ contribution is quite different from the one expected for the decay of a $\rho$ with a Breit-Wigner mass distribution (compare blue and red curves in figure 7 ). This is due to the coupling to the $\mathrm{N}(1520)$ which, according to the preliminary results of the PWA, plays a major role in the off-shell $\rho$ production. Implementing the $\rho$ contribution in the simulation results in a large dilepton yield above 300 $\mathrm{MeV} / \mathrm{c}^{2}$, which improves significantly the description of the data. As shown in figure 4 , the simulation including this $\rho$ contribution is able to reproduce the yield for the exclusive $\pi^{-} \mathrm{p} \rightarrow \mathrm{n} e^{+} e^{-}$reaction. According to the PWA, the dominant resonant contribution to the off-shell $\rho$ production is the $\mathrm{N}(1520)$ resonance. By this procedure, we demonstrate experimentally the validity of the VDM approach to take into account the hadronic structure of the electromagnetic transitions in the region of the $\mathrm{N}(1520)$. We can also notice that the yield for missing masses above $1.1 \mathrm{GeV} / \mathrm{c}^{2}$, where the $\eta$ Dalitz decay dominates is also fairly well reproduced, which confirms the consistency of our participant-spectator model.

In the second approach, we used the model of [26] which was developed for the time-like electromagnetic transition form factors of the N-N(1520) transition. In this model, baryons are described with a quark core and a meson cloud, with parameters fitted to the existing data for the N-N(1520) transition in the space-like region. The predictions of the model for the effective form factor $\mathrm{G}_{T}\left(q^{2}\right)$ have been injected using Eq. 1 in our Dalitz decay simulation. The meson cloud contribution is strongly dominant in the time-like region and leads to an enhancement of the dilepton yield for invariant masses larger than $300 \mathrm{MeV} / \mathrm{c}^{2}$. A detailed comparison with the data is in progress.

\section{Angular distributions}

Further information, in particular on the transverse and longitudinal virtual photon polarization can be obtained from the angular distributions. A convenient parametrization of the amplitudes $|A|$ at a given value of $\mathrm{M}_{e e}$ and emission angle of the virtual photon derives from the density matrix formalism [28]

$$
\begin{aligned}
|A|^{2} \propto \quad 4 k^{2}\left[2 \rho_{00}\right. & \left(1-\cos ^{2} \theta\right)+2 \rho_{11}\left(1+\cos ^{2} \theta\right) \\
+ & 2 \sqrt{2} \sin (2 \theta) \cos \phi \operatorname{Re} \rho_{10} \\
+ & \left.2 \sin ^{2} \theta \operatorname{Re} \rho_{1-1} \cos (2 \phi)\right] .
\end{aligned}
$$

Here, $k^{2}, \theta$ and $\phi$ denote the momentum, polar angle and azimuthal angles of the lepton in the virtual photon reference frame, respectively and $\rho_{00}, \rho_{11}, \rho_{1-1}$ are the three independent density matrix coefficients which can be expressed as a function of the electromagnetic form factors or helicity amplitudes. This relation depends on the spin and parity of the transition. Nevertheless, the connection between $\rho_{11}$ and the transverse $A_{\perp}$ and longitudinal $A_{\|}$amplitudes can be simply written as:

$$
\rho_{11}=\frac{A_{\perp}}{2 A_{\perp}+A_{\|}}
$$




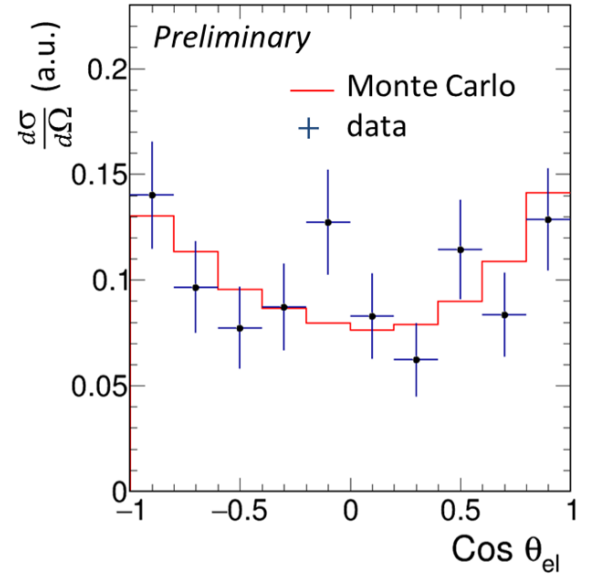

Figure 8. The exclusive $\pi^{-} p \rightarrow n e^{+} e^{-}$reaction is selected using missing mass. The distribution of the lepton angle $\theta_{e l}$ in the $\gamma^{*}$ reference frame is shown for $\gamma^{*}$ angles in the center-of-mass in the bin $0<\cos \left(\theta_{\gamma^{*}}^{C M}\right)<0.5$. The red curve represents the result of the fit weighted by acceptance and efficiency [27].

A method to extract spin density matrix elements from a fit to experimental data has been developed based on the maximum likelihood [29]. The coefficients can be extracted as weights of a Monte-Carlo simulation which takes into account the acceptance and efficiency of the detector, as illustrated in figure 8. Despite the low statistics which affects the precision of the data, the coefficients were studied in three bins of angle of the virtual photon, providing significant information on the spin structure of the transitions. For example, preliminary results indicate that, in some bins $\rho_{11}$ is different from 0.5 and $\rho_{10}$ is different from zero, which clearly demonstrates the contributions of virtual photons with longitudinal polarization, in contrast to real photons.

Moreover, the density matrix formalism can also be applied to analyze the angular distributions of pions from the off-shell $\rho$ component of the PWA solution. The coefficients obtained from the two pion analysis should be consistent with the ones obtained from the dilepton distributions for $M_{e e}>300 \mathrm{MeV}$ if the dominant channel is indeed the $\rho$ meson decay. The studies are on-going and the results are expected soon. The density matrix formalism also allows for an easy comparison with model predictions. As explained in details in D. Nitt's contribution to this conference [30], we used the Lagrangian model of [28], which takes into account VDM form factors for the various baryonic transitions.

\section{Project for future pion beam experiments}

These results from a commissioning experiment provide very first and promising information about the baryon electromagnetic transitions in the time-like region. In the near future, an experiment is planned to explore the third resonance region $(\sqrt{s}=1.7 \mathrm{GeV})$, where several baryonic resonances contribute $(\Delta(1620), \Delta(1700), \mathrm{N}(1650)$,

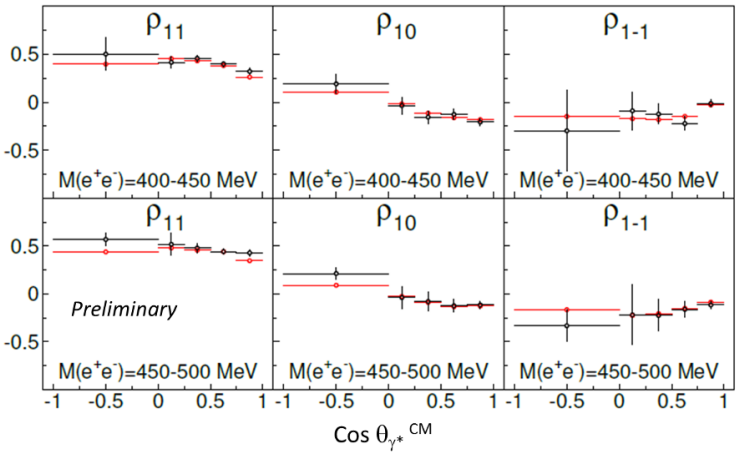

Figure 9. Expected precision on the spin density matrix elements $\rho_{11}, \rho_{10}, \rho_{1-1}$ for $10^{4}$ events as a function of $\mathrm{z}=\cos \theta_{C M}$ and for two bins in dilepton invariant mass $M_{e e}$. The red points show results obtained in the full solid angle and the black points correspond to events measured within the HADES acceptance.

$\mathrm{N}(1675), \mathrm{N}(1630), \mathrm{N}(1710), \mathrm{N}(1720), \ldots)$ [31]. Dielectron, strange and non strange meson production in $\pi^{-} p$ and $\pi^{-} C$ reactions will be measured. In this higher energy range, the sensitivity of electromagnetic form factors to VDM will be enhanced, since $q^{2}$ values closer to the Vector Meson poles can be reached, which will be very valuable e.g. to test predictions of $\rho-\omega$ interferences [32, 33]. Our goal is to reach a statistics for the signal 20 times larger than in the previous commissioning experiment, to allow for an extraction of the global longitudinal (related to the Coulomb form factor) and transverse (related to the electric and magnetic form factors) amplitudes for two bins in invariant mass and three bins in virtual photon angles (see figure 9). The measurements of various exclusive hadronic channels $\left(\pi^{0} \pi^{-} \mathrm{n}, \pi^{+} \pi^{-} \mathrm{p}, \omega \mathrm{n}, \rho \mathrm{n}, \eta \mathrm{n}, \mathrm{K} \Lambda, \mathrm{K} \Sigma\right)$ in an energy scan are also foreseen. The objective is to increase by at least one of order of magnitude the existing data base for these hadronic decay channels. In this way, many poorly known hadronic couplings in the third resonance region could be determined with a precision at the percent level. Many open issues related to baryon structure also exist in this mass region (e.g. $\mathrm{N}(1710), \Delta(1700)$ ) and are in the focus of new meson beam facilities [34]. In relation to the general program of the HADES collaboration, the emphasis for future measurements will be put on improving the knowledge on $\rho-\mathrm{N}, \omega-\mathrm{N}$ and $\mathrm{K}-\Lambda$ couplings, which are needed as input for the modeling of the propagation of $\rho$ mesons and strange particles in matter. The measurement of channels with neutral mesons $\left(\pi^{0}, \eta\right)$ or real photons will be possible thanks to the new Electromagnetic Calorimeter, which has recently been put in operation. A higher electron efficiency is provided by the new RICH photon detector, developed in the context of the HADESCBM FAIR Phase-0 program and which was also recently used successfully by the HADES collaboration in a measurement of $\mathrm{Ag}+\mathrm{Ag}$ collisions. The acceptance will also be improved at the most forward angles $\left(0.6-6.5^{\circ}\right)$ thanks to a new detection system based in particular on straw tube trackers in the context of the HADES/PANDA FAIR Phase-0 program, which is currently under construction. 
We also await improvements of the extraction efficiency of the primary ${ }^{14} \mathrm{~N}$ or proton beams which could result in a significant increase of the pion beam flux.

Measurements on a carbon target, which are needed to extract the proton contribution in the polyethylene target, will also be used to study $e^{+} e^{-}$and meson production in $\pi^{-} C$ reactions. The objective is to pin down possible cold matter effects on the $\rho$ and $\omega$ spectral functions, as well as to quantify $\omega$ absorption.

Further in the future, the very poorly known fourth resonance region could be explored in experiments with a pion beam at $\mathrm{p}_{\pi} \sim 1.3 \mathrm{GeV} / \mathrm{c}$. This kinematical region is well suited to provide accurate measurements of the $\Lambda(1405)$ which can be analyzed both in the $\Sigma \mathrm{N}$ neutral and charged decay modes.

\section{Future studies of hyperon electromagnetic transitions}

The HADES collaboration also plans to investigate the poorly known electromagnetic structure of hyperons. While elastic or transition form factors can be measured at large $q^{2}$ in $e^{+} e^{-}$annihilation reactions [35], the study of Dalitz decay of hyperons $\left(\mathrm{Y}^{\prime} \rightarrow \mathrm{Y}^{+} e^{-}\right)$is unique to investigate the low $q^{2}$ region. One open question which makes studies in this kinematical region very attractive is the relevance of VDM in the hyperon sector [36-38]. Due to the narrow width of most hyperons, they can be reconstructed with the invariant mass of their decay products and one can therefore take advantage of their abundant production in pp reactions (a few hundred $\mu \mathrm{b}$ at $\mathrm{p} \geq 5 \mathrm{GeV} / \mathrm{c}$ ). HADES can study hadronic decays, as well as radiative and Dalitz decays of hyperons. These measurements will also benefit from the on-going upgrade of the HADES set-up. In particular, the above-mentioned increase of the acceptance will be very beneficial for the detection of forward emitted protons. The measurement of radiative decays will also be possible with the new Electromagnetic Calorimeter. The foreseen increase of the acquisition rate (up to $200 \mathrm{kHz}$ ) is also needed to record enough statistics for the rare Dalitz decays. According to the dedicated simulations which have been performed for the $\Lambda(1520) \rightarrow \Lambda$ $e^{+} e^{-}$Dalitz decay, a signal rate of $550 \Lambda e^{+} e^{-}$events per day after all analysis cuts is expected. The comparison of the $N(1520)$ and $\Lambda(1520)$ Dalitz decays both measured with HADES will bring completely new information about the sensitivity of baryon electromagnetic structure to the strange quark content at finite $q^{2}$ in the time-like region. This program will be started at the present SIS18 facility and continued at higher energies at SIS100 (FAIR), in a complementary way to the studies foreseen at PANDA.

\section{Conclusion}

In complement to dense matter studies, the HADES collaboration developed an important program in elementary reactions to shed light on the role of off-shell $\rho$ mesons as a mediator of the electromagnetic interaction. Recently, a new step was taken with an exploratory experiment using the GSI pion beam in the $\mathrm{N}(1520)$ region. It demonstrated the interest of pion beam experiments to perform direct studies of baryon Dalitz decays and to get insight into the completely unknown time-like electromagnetic structure of baryonic transitions at $q^{2}$ below the vector meson poles. The measurement of both $e^{+} e^{-}$and $\pi \pi$ channels allows for detailed checks of Vector Meson Dominance. Such studies will be pursued in the near future with the pion beam at GSI for heavier resonances. In addition, a program focussing on electromagnetic transitions between hyperons will be started at the present SIS18 facility. In further future, the HADES detector will exploit the proton and heavy ion beams of the SIS100 at the FAIR facility and pursue its complementary hadronic physics and dense matter studies at higher energies.

\section{Acknowledgements}

SIP JUC Cracow, Cracow (Poland), National Science Center, 2016/23/P/ST2/040 POLONEZ, 2017/25/N/ST2/00580, 2017/26/M/ST2/00600; TU Darmstadt, Darmstadt (Germany), VH-NG-823, DFG GRK 2128, DFG CRC-TR 211, BMBF:05P18RDFC1; Goethe-University, Frankfurt (Germany) and TU Darmstadt, Darmstadt (Germany), ExtreMe Matter Institute EMMI at GSI Darmstadt; TU München, Garching (Germany), MLL München, DFG EClust 153, GSI TMLRG1316F, BmBF 05P15WOFCA, SFB 1258, DFG FAB898/2-2; NRNU MEPhI Moscow, Moscow (Russia), in framework of Russian Academic Excellence Project 02.a03.21.0005, Ministry of Science and Education of the Russian Federation 3.3380.2017/4.6; JLU Giessen, Giessen (Germany), BMBF:05P12RGGHM; IPN Orsay, Orsay Cedex (France), CNRS/IN2P3; NPI CAS, Rez, Rez (Czech Republic), MSMT LM2015049, OP VVV CZ.02.1.01/0.0/0.0/16 013/0001677, LTT17003.

\section{References}

[1] R. Rapp, J. Wambach, Eur. Phys. J. A6, 415 (1999), hep-ph/9907502

[2] G. Agakichiev et al. (HADES), Eur. Phys. J. A41, 243 (2009), 0902.3478

[3] J. Adamczewski-Musch et al. (HADES), Nature Phys. 15, 1040 (2019)

[4] P. Salabura et al. (HADES), Exploring time like transitions in $p p, \pi^{-} p$ and heavy ion reactions with HADES, NSTAR2019

[5] I. Ciepal et al. (HADES), Partial wave analysis of hades data for two-pion production in pion- nucleon reactions, NSTAR2019

[6] V. Burkert, $N^{*}$ Experiments and their Impact on Strong $Q C D$, NSTAR2019

[7] J. Adamczewski-Musch et al. (HADES), Phys. Rev. C95, 065205 (2017), 1703.07840

[8] M. Tanabashi et al. (Particle Data Group), Phys. Rev. D 98, 030001 (2018)

[9] G. Agakishiev et al. (HADES), Phys. Rev. C85, 054005 (2012), 1203.2549 
[10] G. Agakishiev et al. (HADES), Eur. Phys. J. A48, 64 (2012), 1112.3607

[11] G.. Agakishiev et al. (HADES), Eur. Phys. J. A50, 82 (2014), 1403. 3054

[12] R. Simon et al., Prog. Part. Nucl. Phys. 247, 42 (1999)

[13] J. Diaz et al., Nucl. Instrum. Meth. A478, 511 (2002)

[14] CNS Data Analysis Center, SAID Home Page, http://gwdac.phys.gwu.edu/

[15] A. Anisovich et al., Eur. Phys. J. A24, 111 (2005), hep-ph/0407211

[16] F. Scozzi (HADES), EPJ Web Conf. 137, 05023 (2017)

[17] I. Fröhlich, F. Dohrmann, T. Galatyuk, R. Holzmann, P. Kahlitz et al., Eur. Phys. J. A45, 401 (2010), 0909.5373

[18] F. Bulos et al., Phys. Rev. Lett. 13, 558 (1964)

[19] A. Starostin et al., Phys. Rev. C72, 015205 (2005)

[20] R.A. Burnstein, G.R. Charlton, T.B. Day, G. Quareni, A. Quareni-Vignudelli, G.B. Yodh, I. Nadelhaft, Phys. Rev. 137, B1044 (1965)

[21] A.D. Brody et al., Phys. Rev. D4, 2693 (1971), [Erratum: Phys. Rev.D5,2899(1972)]

[22] S. Prakhov et al. (Crystal Ball Collaboration), Phys.Rev. C69, 045202 (2004)

[23] S. Prakhov et al., Phys. Rev. C72, 015203 (2005)

[24] I. Fröhlich et al., PoS ACAT2007, 076 (2007), 0708.2382
[25] M.I. Krivoruchenko, A. Faessler, Phys. Rev. D65, 017502 (2002)

[26] G. Ramalho et al., Phys. Rev. D93, 033004 (2016), 1512.03764

[27] B. Ramstein et al. (HADES), EPJ Web Conf. 199, 01008 (2019)

[28] E. Speranza, M. Zetenyi, B. Friman, Phys. Lett. B764, 282 (2017), 1605 . 04954

[29] V.V. Sarantsev, private communication

[30] D. Nitt, Dilepton production and anisotropy in pionnucleon collisions, NSTAR2019

[31] Proposal for experiments at SIS18 during FAIR Phase0, Properties of hadron resonances and baryon rich matter, (HADES Collab.), GSI 2017, http://repository.gsi.de/record/220071/files/HADES

[32] A.I. Titov, B. Kämpfer, Eur. Phys. J. A12, 217 (2001), nucl-th/0108019

[33] M. Lutz, B. Friman, M. Soyeur, Nuclear Physics A 713, 97 (2003.)

[34] W.J. Briscoe et al., Eur. Phys. J. A51, 129 (2015), 1503.07763

[35] K. Schonning, Hyperon physics with PANDA at FAIR, NSTAR2019

[36] R.A. Williams, C.R. Ji, S.R. Cotanch, Phys. Rev. C48, 1318 (1993)

[37] G. Ramalho, K. Tsushima, Phys. Rev. D88, 053002 (2013), 1307.6840

[38] C. Granados, S. Leupold, E. Perotti, Eur. Phys. J. A53, 117 (2017), 1701.09130 